\title{
Review of: "Accuracy of High-risk HPV DNA PCR, p16INK4a Immunohistochemistry or the Combination of Both to Diagnose HPV-driven Oropharyngeal Cancer"
}

\author{
Sveinung Wergeland Sørbye
}

Potential competing interests: The author(s) declared that no potential competing interests exist.

About 1 in 3 cases of oropharyngeal cancer is caused by human papillomavirus (HPV). Patients with HPVinduced cancer respond better to treatment and have a better prognosis than patients with oropharnx cancer caused by tobacco and alcohol. This means that the treatment can be adapted to each individual patient based on HPV status. It is important to have reliable and accurate biomarkers to diagnose truly HPV-induced oropharyngeal cancer.

Both HPV testing and immunostaining with p16 have been used to distinguish HPV-induced cancer from other cancers. Simoens et al. have explored the accuracy of three possible test strategies, HPV DNA test alone, p16 immunohistochemistry alone and the combination of both tests, considering double positivity (p16+/HPV+) as positivity criterion. They used tissue samples from 99 patients with oropharyngeal cancer. Presence of HPV E6*I mRNA was considered as the gold standard, indicating HPV etiology.

The detection rates of DNA, p16 and mRNA were $36.4 \%, 34.0 \%$ and $28.9 \%$. Using mRNA status as the reference, DNA testing showed $100 \%$ (28/28) sensitivity, and $92.5 \%(62 / 67)$ specificity for the detection of HPV-driven cancer. The sensitivity and specificity of combined p16+DNA testing was $96.4 \%(27 / 28)$ and $97.0 \%(65 / 67)$.

Since HPV-driven oropharyngeal cancer can be treated less aggressively, combined testing (p16+DNA) can increase specificity and reduce treatment sequelae. Combined testing enhances specificity up to $97 \%$, while maintaining high sensitivity (96\%), compared to single testing. Treatment adjustments can be encouraged based on this combined test result.

The claims are properly placed in the context of the previous literature. The experimental data support the claims. The manuscript is written clearly enough that most of it is understandable to non-specialists. The authors have provided adequate proof for their claims, without overselling them. The authors have treated the previous literature fairly. The paper offers enough details of methodology so that the experiments could be reproduced. I liked the article. 
